\title{
Towards a simulation-based framework for evaluating energy-efficient solutions in train operation
}

\author{
V. de Martinis ${ }^{1}$, U. Weidmann ${ }^{2}$ \& M. Gallo ${ }^{3}$ \\ ${ }^{1}$ Dipartimento di Ingegneria Civile Edile e Ambientale, \\ Università degli studi di Napoli Federico II, Italy \\ ${ }^{2}$ Institut für Verkehrsplanung und Transportsysteme, \\ ETH Zurich, Switzerland \\ ${ }^{3}$ Dipartimento di Ingegneria, Università del Sannio, Italy
}

\begin{abstract}
In this paper we propose a simulation-based framework for evaluating energyefficient solutions in train operation. The general framework is composed of an optimisation system able to generate energy-efficient station-to-station speed profiles, looped with a micro-simulation tool for simulating railway traffic conditions, in order to evaluate the impacts on railway systems (delays, conflicts) and energy savings. The optimisation system is a subroutine consisting of a Genetic Algorithm for optimal speed profile parameters optimisation, a speed profile generator, and an energy consumption model. The micro simulation tool allows the evaluation of the impact of energy efficient speed profiles on rail operation. The framework operates on a database composed of 4 subsets: timetable, rolling stock characteristics, signalling system, infrastructure features; the first subset can be considered as the result of scheduling or rescheduling procedures, while the others can be assumed to be fixed. The proposed framework has been applied on a real-scale case of an Italian suburban railway system.
\end{abstract}

Keywords: energy saving, speed profile, simulation. 


\section{Introduction and literature review}

Energy efficiency in railway systems has rapidly become a hot issue in railway systems, involving both the academic world and industry.

In the wide literature of the field it is possible to identify several approaches for defining optimal energy efficient solutions. Regarding the level of control it is possible to highlight two different classes: the rail traffic control and the train operation control. Although the rail traffic control is mainly focused on conflicts avoidance, delay reduction and, in general, maximum exploitation of the system, in many cases solutions are also optimised for energy consumption reduction (see D'Ariano et al. [1]; Corman et al. [2]; Rao et al. [3]). The train operation control is focused on single train dynamics and an energy efficient solution can be directly developed through an optimization of train trajectories or speed profiles. On the other hand, train operation control does not provide the system with information on the whole network. In this level of control there are many approaches that have been developed during the years. A widely studied approach for energy saving involves formulation of an optimal control problem by applying Pontryagin's maximum principle (see Hansen and Pachl [4] for a description) in order to obtain optimal train operation regimes. The problem has been specified for different control cases (discrete, continuous) and operation conditions (Howlett [5]; Khmelnitsky [6]). By applying a dynamic programming approach, the optimisation problem can be decomposed into several simpler subproblems and solved with recursive methods. Some major results have been shown through the definition of a multi-stage decision process by Albrecht and Oettich [7], Franke et al. [8] and Ko et al. [9] for optimisation of the reference trajectory. Some other approaches refer to a direct model formulation that leads to a nonlinear problem resolution through different algorithms and optimization procedures (see for example Wang et al. [10]).

In the last few years, with the constant development of simulation tools, the number of simulation-based approaches has increased. Thanks to the undisputed advantages of simulation models, it is possible to find optimal solutions by estimating the control parameters that better fit the requested needs, following the What If planning approach (see Cascetta [11] for description). Some examples of successful adoption of simulation-based optimisation procedures are given by Quaglietta et al. [12] where a parallel computing approach was applied on an optimisation loop, comprising an optimisation algorithm and a simulation tool, so as to obtain significant results in terms of computing time and some significant applications on the speed profile effects such as quality of service and travel demand costs (D'Acierno et al. [13]). Corapi et al. [14] and De Martinis et al. [15] proposed to adopt a microscopic approach for analysing effects of different driving strategies in terms of energy consumption.

In this paper, formulations and constraints of the applied models are described in section 2, the description of the simulation-based framework is proposed in section 3, the application of a real case is provided in section 4, together with final considerations and further development in section 5 . 


\section{Formulation of models}

\subsection{Speed profile definition}

A train speed profile can be modelled as the result of the efforts applied at the wheels from the train motion system (i.e. traction units plus braking system), vehicle resistances and line resistances. By analysing the single components of the differential equation of the motion (1), the relationship between vehicle dynamics (that include the rotating masses factor $\gamma$ ), applied efforts $(F)$ and resistances $(R)$ :

$$
m \cdot \gamma \cdot \mathrm{d} v / \mathrm{d} t=F(v)-R(v, s) .
$$

is constrained by train characteristics (i.e. available power, allowable adherence on rail, etc.) and service requirements (i.e. allowable acceleration and deceleration rates for passenger comfort, etc.). The term $R$ considers both vehicle resistances (that depend on speed $v$ ) and line resistances (that depend on train position along the track $s$ ). In many cases, eqn (1) is solved by a finite difference method for a single time interval $(i, i+1)$ :

$$
m \cdot \gamma \cdot \frac{\Delta v}{\left(t_{i+1}-t_{i}\right)}=F\left(v_{i}\right)-R\left(v_{i}\right)-R\left(s_{i}\right) .
$$

where the tractive effort depends on the train speed and the characteristics of the traction unit, and the line resistances are computed considering the train position at interval $i$. The related constraints on train characteristics are given by:

$$
F\left(v_{i}\right) \leq F_{\max }\left(v_{i}\right)=k_{0, k}+k_{1, k} \cdot v+k_{2, k} \cdot v^{2} .
$$

where the $k$ coefficients are specified for the single train and usually given by the train builder. Regarding train dynamics, the following constraints are considered:

$$
\begin{aligned}
& \frac{\Delta v}{\left(t_{i+1}-t_{i}\right)} \leq a_{\max } \\
& \frac{\Delta v}{\left(t_{i+1}-t_{i}\right)} \geq d_{\max } \\
& v_{i} \leq v_{\max , i}(s) .
\end{aligned}
$$

Eqns (4) and (5) are related to comfort limits of acceleration and deceleration phases respectively, while equation (6) ensures the respect of speed limits along the track.

Speed profiles can be defined through motion parameters such as acceleration and braking rates, cruising speeds and their related switching points. Whereas the $F\left(v_{i}\right)$ is strictly less than its maximum values, as shown in eqn (3), traction units 
can follow the given acceleration rate, while in case of $F\left(v_{i}\right)$ equal to the $F_{\max }\left(v_{i}\right)$ value, acceleration is driven by the traction unit performances. Cruising speed assumes that efforts given by the train motion system must have the same absolute value of resistances at that speed and in according with eqn (3). Braking rates are mostly constrained by adherence values and, although braking profiles are the subject of different studies, in this paper we refer to general values of braking rates both in terms of emergency braking and in terms of comfort braking; these values are quite restrictive, ensuring adherence conditions at different speeds but extending the estimated braking space. Moreover, the studies of specific speed profiles for energy saving often include a coasting phase, that consists of switching off the engine at a given time or position and letting the train run spending its acquired kinematic energy; in this motion phase, train dynamics are driven by vehicle and line resistances. The switching points of the coasting phase can be computed after defining the other variables and according to a specific strategy; starting from a given switching point, the speed $v_{i+1}$ can be computed according to eqn (2) in order to estimate the next step resistances. The end switching point of the coasting phase is computed according to speed restrictions and applied braking rate.

In this paper we consider an acceleration function, introducing the variation of acceleration $\Delta a\left[\mathrm{~m} / \mathrm{s}^{3}\right]$ that allows us to simulate transitions between two consecutive motion phases. This parameter can be considered as a behavioural aspect that belongs to train driving (specific train driver attitude) and it can be quite difficult to calibrate; on the other hand, if driverless systems or the latest driving assistance systems on board are considered, this parameter can also be taken into account in some optimisation procedures. The parameter $\Delta a$ has not been considered for those cases in which variation of acceleration is driven by traction unit performances (from acceleration to cruising when $F=F_{\max }$ ) or driver's action let the transition be quite fast (engine off for coasting). For our purposes, $\Delta a$ will be assumed to be fixed with a value of 0.5 .

\subsection{Timetable constraints definition}

In order to define an acceptable speed profile, the scheduled timetable and the distance to cover have to be considered. Because timetable definition can follow different rules in accordance with different types of service, it can be assumed that the relationship between the estimated arrival time and the scheduled arrival time of a train at a generic station $J$, is:

$$
\hat{T}_{a r r, J} \leq T_{a r r, J}
$$

and that the obvious relation between the space covered $S$ with the proposed speed profile $S P$ and the distance between station $J-1$ and $J$ is satisfied:

$$
S\left(S P_{J-1, J}\right)=\operatorname{Dist}(J-1, J)
$$


In practice, the scheduled timetable is composed of minimum service times, typically minimum headways, minimum running times and minimum dwelling times, and their related recovery times. Recovery times can be classified in time for reducing small delays of trains (running time reserve, dwell time reserve) and time for avoiding delay propagation between different trains (buffer time). Where it is possible, these times are usually considered as times available for implementing energy efficiency strategies. In planning processes, recovery times can lead to improvements in both train punctuality and timetable stability (Goverde [16]), although their use should consider the specificity of the service on which they are applied (D'Ariano et al. [1]).

According to eqn (7), the $T_{a r r, J}$ at station $J$ is computed by considering the scheduled train departure from the previous station $J-1$, the minimum running time and the running time reserve. Energy efficiency strategies can be adopted when the expected delay $D_{J-1, J}$ during the journey is lower than the related running time reserve $R T R_{J-1, J}$ :

$$
D_{J-1, J}<R T R_{J-1, J}
$$

When the delay increases, the reduction of quality of service must be minimised, and this means that a time-optimal driving strategy must be adopted (maximum feasible values of acceleration, deceleration and cruising speed), while energy saving strategies cannot be considered. In the same way, dwell time reserves $(D T R)$ at stations and buffer times (BT) (see Hansen and Pachl [4]) can also be considered for implementing energy efficiency driving strategies. In this paper we consider only the running time reserve as extra time for implementing energy efficient driving strategies.

\section{The simulation based framework}

The proposed simulation-based framework has been designed in order to provide a useful evaluation tool for an energy efficient driving solution. In this paper, for energy efficient driving solutions we refer to energy efficient speed profiles that allow us to minimise energy consumption given the requested quality of service. The main aim is to provide the train operators and the rail managers with additional information both during train operation and for planning purposes.

The simulation-based framework consists of a closed loop, described in De Martinis et al. [15], and is composed of a speed profile optimization tool, a simulation tool, and a database with information about timetable, rolling stock characteristics, infrastructure details and signalling system features. The framework is reported in Figure 1.

The database contains information for both the simulation tool and the optimization tool. More precisely, rolling stock, signalling system and infrastructure data can be assumed as defined by the user, while the timetable can either represent a specific operating scenario or be the result of rescheduling procedures, e.g. rescheduling for conflicts resolution. 
The database also includes information on real train trajectories, such as arrivals and departures, actual reserve times and energy consumptions. If some data are missing, it can be useful to evaluate them with a calibrated simulation tool.

The speed profile optimisation tool is composed of the optimisation algorithm, the speed profile generator and the energy consumption model.

The chosen optimisation algorithm is a Genetic Algorithm that allows fast response and good quality for finding good local minima. The input of the GA is the energy consumption retrieved for the given station to station speed profile, while the outputs are the motion parameters, i.e. acceleration rate, deceleration rate and the cruising speeds. Solutions are constrained by eqns (4), (5) and (6). A complete formulation of the optimisation problem can be found in De Martinis et al. [15].

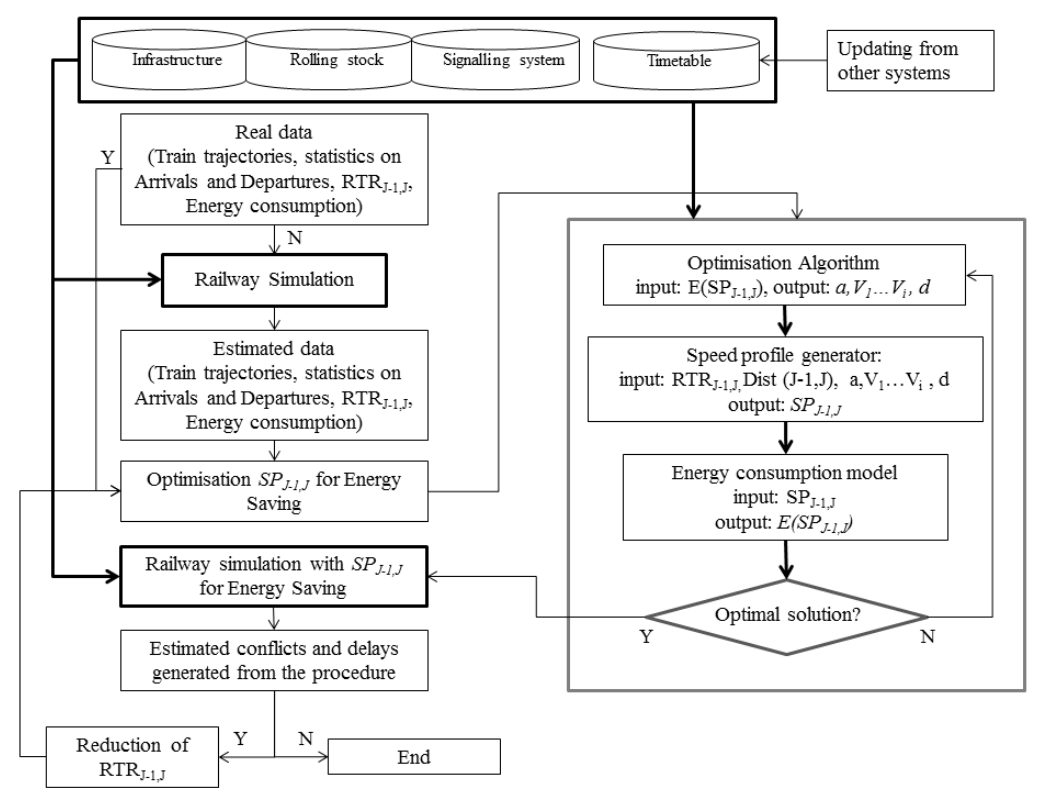

Figure 1: The adopted framework for energy saving speed profile definition.

The speed profile generator provides the station to station speed profile according with the motion parameters generated by the GA, database and real time information, and according with the selected driving strategy (e.g. coasting, no coasting), so defining the switching points between two consecutive motion phases. For a given coasting strategy, the speed profile generator verifies the consistency of the profile in terms of travel time available on the given track and the distance covered, i.e. constraints (7) and (8), using the motion parameters generated by the optimisation algorithm. In this paper we use the strategy ASAP (As Soon As Possible), which means that the driver starts coasting as soon as the condition allow to respect condition (7) and (8). Moreover, coasting will be used 
only before the final brake. Actually, the vector of optimised speed profile parameters for a station to station track $J-1, J$ is composed by:

$$
S P_{J-1, J}=S P_{J-1, J}\left(a^{*}, v_{1}{ }^{*}, \ldots, v_{i}{ }^{*}, T_{i C}{ }^{*}, T_{f C}{ }^{*}, d^{*}\right) \text {. }
$$

The energy consumption model is defined following a discrete approach. The single train energy consumption can be computed as follows:

$$
E=\sum_{i=1 \ldots T} V_{i} \cdot F_{i}^{+}\left(V_{i}, t_{i}\right) .
$$

Energy consumption refers to the positive values of the effort applied at the wheels, i.e. tractive effort during acceleration and cruising. The parameters of the most efficient speed profile so defined are the input of the simulation tool that verifies the impact on the entire network. If new delays and/or conflicts are generated, restrictions on the use of $R T R_{J-1, J}$ have to be applied.

The simulation tool is a microscopic synchronous tool that is able to reproduce and elaborate the behaviour and the performances of all railway elements: infrastructure, signalling systems, rolling stock and timetable. The outputs of the speed profile optimisation loop are included in the simulation scenario of the rail network. Results after simulation are the trains' trajectories, together with their blocking time diagrams and possible conflicts, and energy consumptions.

\section{A test on a real case: the Cumana line}

The Cumana line is a $20 \mathrm{~km}$ suburban line that operates in the west part of Napoli, Italy, connecting the town of Torregaveta, the town of Pozzuoli and the city centre, with several stops in main streets of Napoli and between these two towns. Before its incorporation into a public holding, the former owner was both the track owner and the only operator of this line; nowadays the Cumana line is the only line that operates on this infrastructure.

This application focuses on a hypothetical planning case, the aim of which is to optimise trains speed profiles for energy saving with the existing rolling stock, infrastructure, signalling system and timetable. In order to estimate the available time and to implement speed profiles for energy saving, information about real train trajectories and real departures and arrivals are needed. At this time, this information is partially retrievable, so an already calibrated model has been used for integrating the dataset.

In Figure 2 it is shown the development of the line, together with the stops, and the declared line services between 10:00am and 11:00am. In red are reported the simulation results of the line services in the same time graph.

For a better understanding of the process, a specific ride, which is identified with the code "Cumana MS-TG.7", has been considered. Simulation outputs have shown that, in particular, the arrivals in Corso Vittorio Emanuele and in Fusaro, with a time optimal speed profile, are more than 60s (respectively 69s and $85 \mathrm{~s}$ ) earlier than the planned arrival time, so it is possible to adopt an optimised speed profile for energy saving. In Figure 3, the speed distance diagram of "Cumana MS-TG.7" is reported. 


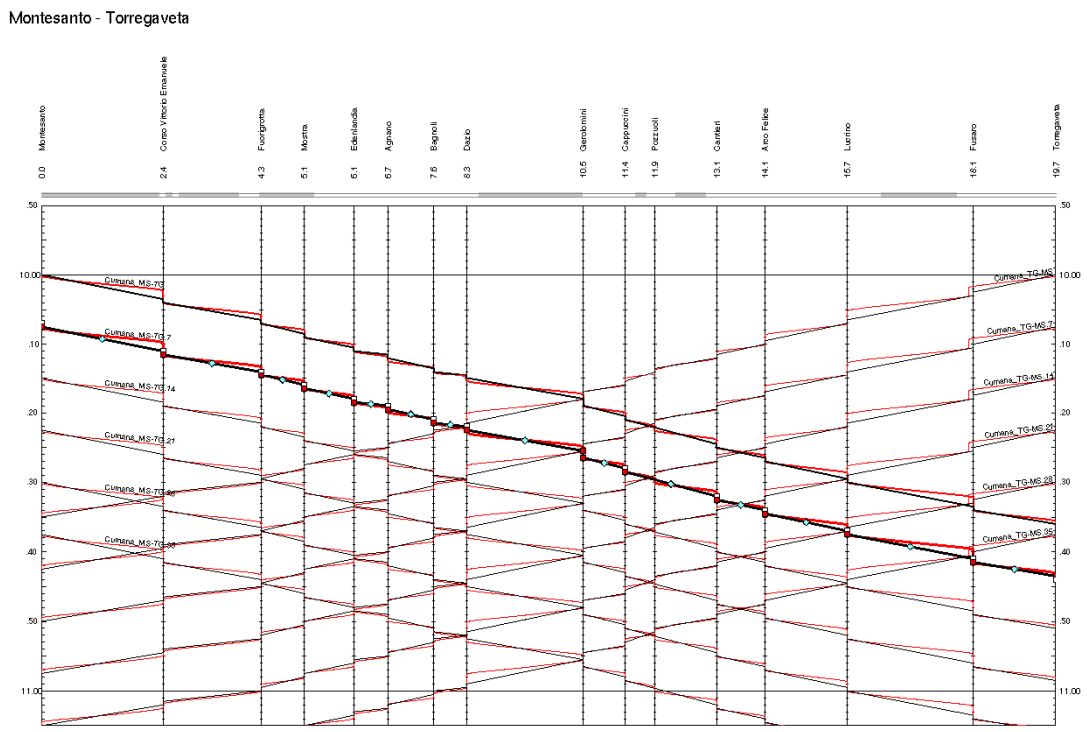

Figure 2: Time graph of the Cumana line with the declared (black) and estimated (red) line running times.

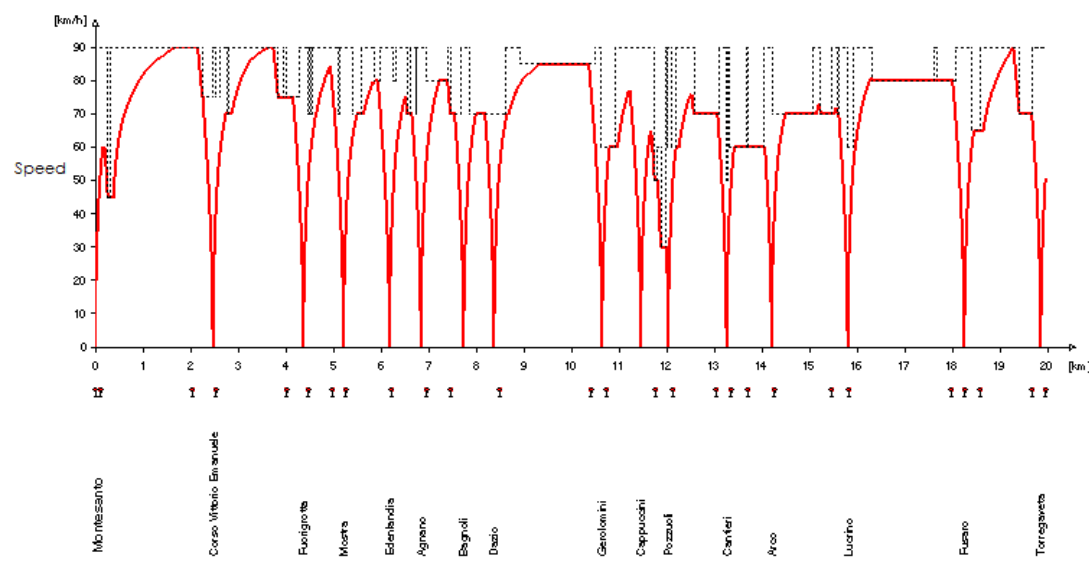

Figure 3: Speed distance graph in time optimal simulated regime.

Optimisation procedures have been considered on the following station to station track: the Montesanto-Corso Vittorio Emanuele and the Lucrino-Fusaro.

The extra time available has not been totally considered, because there was not enough information on the random aspects of the service. Previous studies showed that the average delay is mostly conditioned by the randomness of the dwell time (Quaglietta et al. [12]; Corapi et al. [14]). 
Taking into consideration the current literature and the purposes of this paper, the $30 \%$ of the estimated time available has been used for energy saving driving strategies. Optimisation results are reported in Table 1.

Table 1: Optimized speed profile parameters for energy saving.

\begin{tabular}{|cccccccccc|}
\hline MS-CVE & $\mathbf{J}$ & Acc. & $\mathbf{V}_{\mathbf{1}}$ & $\mathbf{V}_{\mathbf{2}}$ & $\mathbf{V}_{\mathbf{3}}$ & $\mathbf{T}_{\mathrm{ic}}$ & $\mathbf{T}_{\mathrm{fc}}$ & Dec. & Total time \\
\hline T.O. & $/$ & 0.9 & 60 & 45 & 90 & $/$ & $/$ & 0.9 & 142 \\
E.S. & 0.6 & 0.842 & 61.535 & 45.1 & 68.8 & 122 & 143 & 0.894 & 161 \\
& & & & & & & & & \\
& LU-FUS & $\mathbf{J}$ & Acc. & $\mathbf{V}_{\mathbf{1}}$ & $\mathbf{T}_{\mathrm{ic}}$ & $\mathbf{T}_{\mathrm{fc}}$ & Dec. & Total time & \\
\cline { 2 - 8 } & T.O. & $/$ & 0.9 & 80 & $/$ & $/$ & 0.9 & 135 & \\
E.S. & 0.6 & 0.837 & 72.9 & 81 & 147 & 0.887 & 163 & \\
\hline
\end{tabular}

The energy saving speed profiles have been built taking into consideration the infrastructure layout, signalling system and rolling stock characteristics.

The output of the speed profile generator has been generated in accordance with the simulator output format, which defines for each time step (1 second) the speed profile parameters, the requested power and the energy consumption. Optimised speed profiles are shown in Figure 4.

The simulation tool has been set in accordance with the new speed profile parameters on the selected station-to-station tracks. Results are shown in terms of a speed distance diagram and an energy consumption diagram (Figure 5). A train graph with the new speed profile is very similar to the one shown in Figure 2, due to the scale factor, so it is not reported due to this lack of clarity. In any case, conflicts or delays were not generated from the simulation output.

The total amount of energy saved is about $48 \mathrm{MJ}$ (equivalent to $13.34 \mathrm{kWh}$ ) for a total of $604.06 \mathrm{MJ}$ (equivalent to $167.8 \mathrm{kWh}$ ), spent with time optimal driving strategies, that means a reduction of about $8 \%$ of the total energy spent.

\section{Conclusions and further developments}

In this paper we focused on the adoption of simulation tools for evaluating energy saving speed profiles in terms of energy consumption reduction and impact on line services. In particular, some conclusions can be seen from the application phase and the results. The database used for energy saving speed profile definition should contain information about the real speed profiles and the real arrivals and departures; otherwise, information from a calibrated simulation tool has to be taken into consideration. The same consideration can be made for information about energy consumption. It should always be clear that using simulation data on speed profiles, even on a calibrated model, is purely indicative, and that for evaluating the impact on rail services it could be enough to consider a calibrated model on real departures and arrivals, while for the evaluation of energy consumption differences between two different speed profiles, it could be better to evaluate in terms of percentages. As is shown in the previous sections, and as is pointed out in many papers of the current literature, a single simulation shot refers to a particular condition, for example an ideal condition, that may not be reached during real operating conditions. 


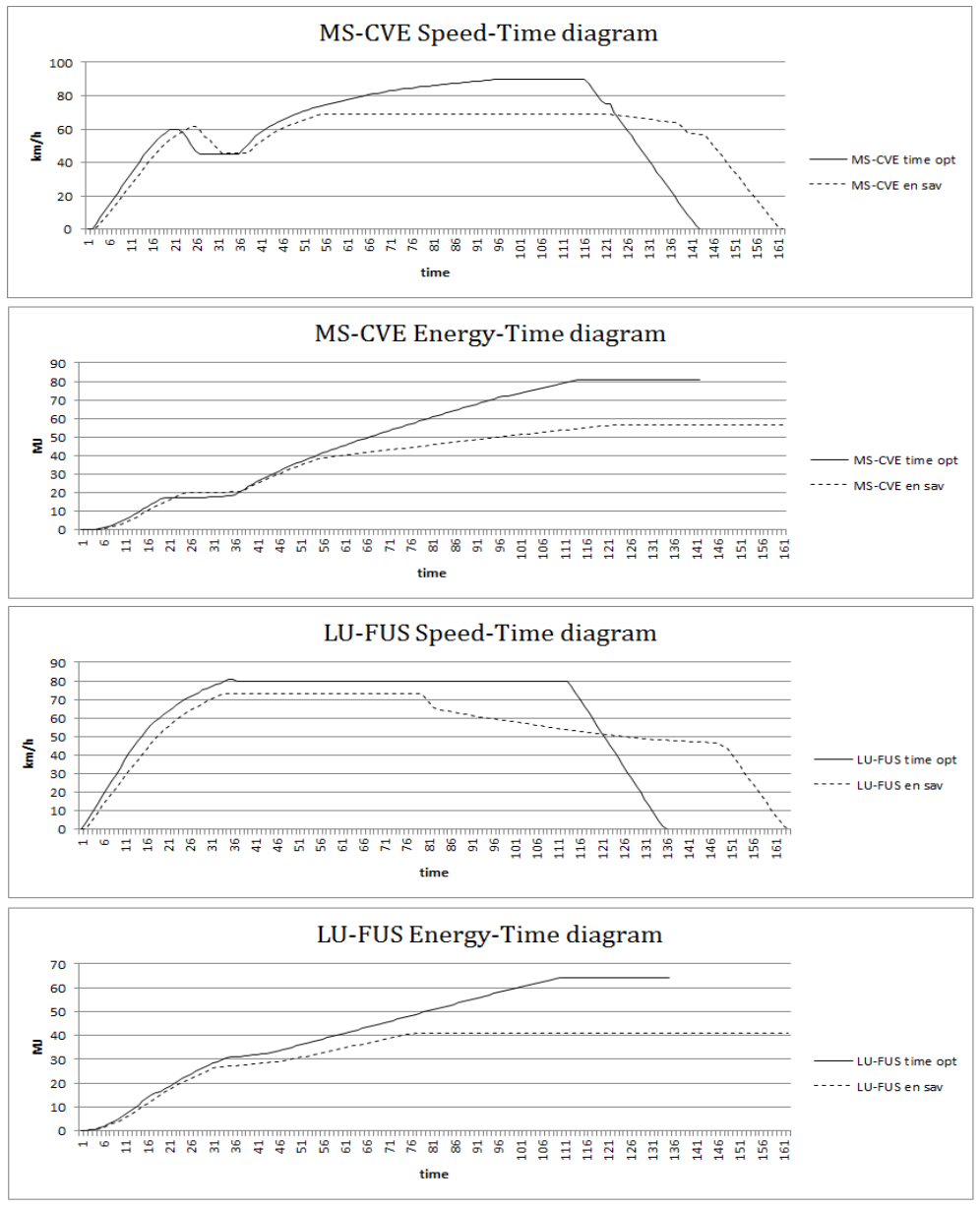

Figure 4: Optimised speed profiles for energy savings.

This means that multiple simulation shots are needed in order to evaluate the distribution of arrivals and departures, for rail impact evaluation, and the distribution of energy consumptions between two different speed profiles.

The results of a single simulation shot have been provided here taking into consideration the randomness of the events estimated in other papers, and reducing the extra time availability for energy saving.

The speed profile generator must be built, in accordance with the features of the chosen simulation tool, in order to have an easy data exchange. It is also possible to set up an optimisation tool which directly builds speed profiles with the simulation tool, but the first impressions of the authors are that the procedure can take a lot of time if some assumptions and simplifications are not taken into account; moreover for real time applications, an estimation of the further arrival 


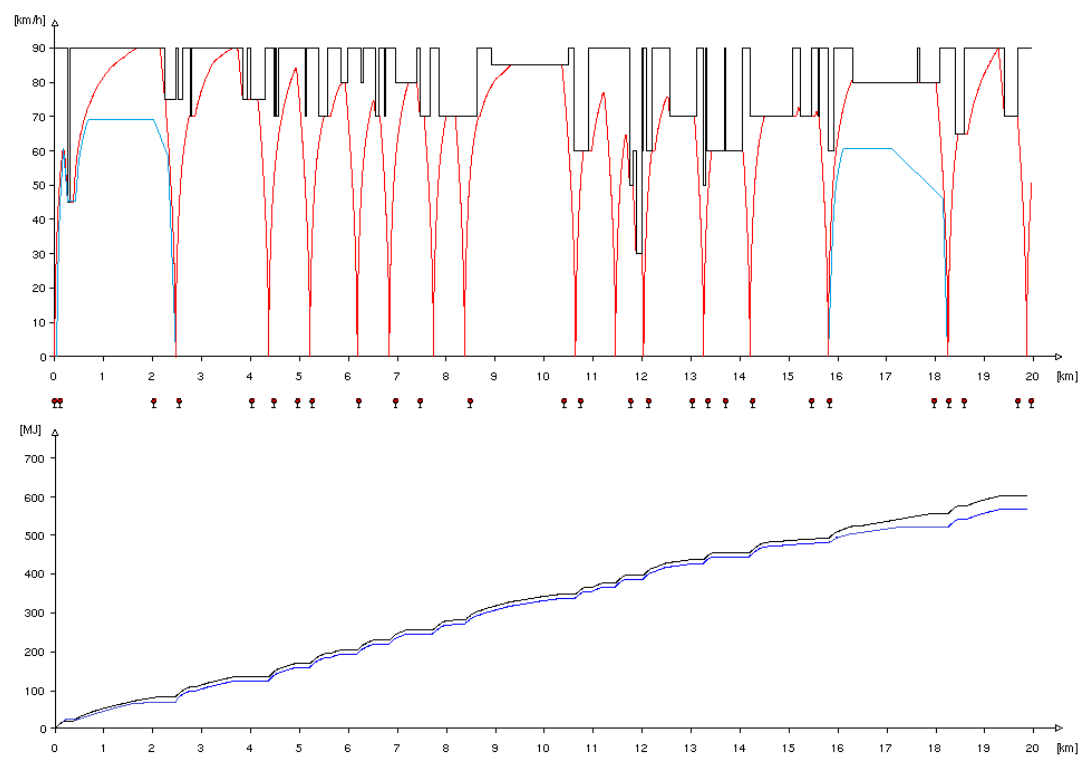

Figure 5: Speed distance graph and energy distance graph considering time optimal speed profile and energy saving speed profile (in blue).

and departure time is needed (e.g. via simulation) and the optimisation of fewer parameters (i.e. cruising speed and switching points of the coasting phase) can ensure a reduction in the computing time.

This test shows that it is possible to build an integrated tool for both defining an optimised train speed profile via simulation and evaluating the impact on the whole service. Further research will be done to implement the framework to the evaluation of distributions of both arrivals and departures and energy consumption, moreover different numbers of speed profile parameters will be tested in order to evaluate the ones that better fit real time requirements.

\section{Acknowledgement}

Partially supported under research project PON - SFERE grant no. PON01_00595.

\section{References}

[1] D'Ariano, A., Pacciarelli, D., \& Pranzo, M., Assessment of flexible timetables in real-time traffic management of a railway bottleneck. Transportation Research Part C, 16, pp. 232-245, 2008. 
[2] Corman, F., D'Ariano, A., Pacciarelli, D., \& Pranzo, M. (2009). Evaluation of green wave policy in real-time railway traffic management. Transportation Research Part C, 17, pp. 607-616, 2009.

[3] Rao, X., Montigel, M. \& Weidmann, U., Methods to improve railway capacity by integration of automatic train operation with centralized traffic management. In: Proceedings of the 5th International Seminar on Railway Operations Modelling and Analysis - RailCopenhagen 2013, 2013.

[4] Hansen, I., \& Pachl, J., Railway, Timetable \& Traffic: Analysis, Modelling, Simulation, Eurailpress: Hamburg, Germany, 2008.

[5] Howlett, P., The optimal control of a train. Annals of Operations Research, 98, 65-87, 2000.

[6] Khmelnitsky, E., On an optimal control problem of train operation. IEEE Transactions on Automatic Control, 45, 1257-1266, 2000.

[7] Albrecht, T., \& Oettich, S., A new integrated approach to dynamic schedule synchronization and energy saving train control. Computer in Railways VIII eds. J. Allan, R. J. Hill, C. A. Brebbia, G. Sciutto \& S. Sone, WIT Press: Southampton, United Kingdom, pp. 847-856, 2002.

[8] Franke, R., Terwiesch, P., \& Meyer, M., An algorithm for the optimal control of the driving of trains. Proceedings of the 39th IEEE Conference on Decision and Control, Sydney, Australia, pp. 2123-2128, 2003.

[9] Ko, H., Koseki, T., \& Miyatake, M., Application of dynamic programming to optimization of running profile of a train. Computer in Railways $I X$, eds. J. Allan, C. A. Brebbia, G. Sciutto \& S. Sone, WIT Press: Southampton, United Kingdom, pp. 103-112, 2004.

[10] Wang, Y., De Shutter, B., Van der Boom, T. J. J., \& Ning, B., Optimal trajectory planning for trains - A pseudospectral method and a mixed integer linear programming approach. Transportation Research Part C, 29, pp. 97-114, 2013.

[11] Cascetta E., Transportation system analysis: Models and applications, Springer: New York, 2009.

[12] Quaglietta, E., D’Acierno, L., Punzo, V., Nardone, R. \& Mazzocca, N., A simulation framework for supporting design and real-time decisional phases in railway systems. Proc. of the 14th IEEE Conference on Intelligent Transportation Systems (ITSC), pp. 846-851, 2011.

[13] D'Acierno, L., Gallo, M., Montella, B. \& Placido, A., Analysis of the interaction between travel demand and rail capacity constraints. WIT Transactions on the Built Environment, 128, pp. 197-207, 2012.

[14] Corapi, G., Sanzari, D., De Martinis, V., D’Acierno, L. \& Montella, B., A simulation-based approach for evaluating train operating costs under different signalling systems. WIT Transactions on the Built Environment, 130, pp. 149-161, 2013.

[15] De Martinis, V., Gallo, M. \& D'Acierno, L., Estimating the benefits of energy-efficient train driving strategies: a model calibration with real data. WIT Transactions on the Built Environment, 130, pp. 201-211, 2013.

[16] Goverde, R. M. P., A delay propagation algorithm for large-scale railway traffic networks Transportation Research Part C, 18, pp. 269-287, 2010. 